\title{
Dynamical Phase Transitions in the Two-Dimensional ANNNI Model
}

\author{
Michael N. Barber ${ }^{1,2}$ and Bernard Derrida ${ }^{1,3}$
}

Received December 8, 1987

\begin{abstract}
We study the phase diagram of the two-dimensional anisotropic next-nearest neighbor Ising (ANNNI) model by comparing the time evolution of two distinct spin configurations submitted to the same thermal noise. We clearly see several dynamical transitions between ferromagnetic, paramagnetic, antiphase, and floating phases. These dynamical transitions seem to occur rather close to the transition lines determined previously in the literature.
\end{abstract}

KEY WORDS: Dynamics; ANNNI model; Monte Carlo.

\section{INTRODUCTION}

Finding the phase diagrams of systems with competing interactions is known to be a difficult problem in statistical mechanics. The main difficulties arise since it is not known a priori whether the system can order at low enough temperature and, if it does, how many pure states characterize the ordered phase. Because of these difficulties spin glasses ${ }^{(1,2)}$ and models such as the axial next-nearest neighbor Ising (ANNNI) model ${ }^{(3,4)}$ have been the subject of controversy during the past decade.

Recently, a new dynamical method ${ }^{(5)}$ for probing phase diagrams has been proposed based on a comparison of the time evolution of distinct configurations subjected to the same thermal noise. The main idea is the following: in a high-temperature phase, thermal noise will be strong

\footnotetext{
'Institute for Theoretical Physics, University of California, Santa Barbara, California 93106.

${ }^{2}$ Permanent address: Department of Mathematics, The Faculties, Australian National University, Canberra, ACT 2601, Australia.

${ }^{3}$ Permanent address: Service de Physique Théorique, CEN Saclay, F-91191 Gif-sur-Yvette, France.
} 
enough to make the system forget its initial configuration. Consequently, one expects that, in a paramagnetic phase, two different configurations will end up becoming identical. On the contrary, in a low-temperature phase, there will exist a finite probability that different initial configurations will fall into different pure phases. Hence, by looking at the time evolution of the distance between two configurations submitted to the same thermal noise, one can hope to determine the phase diagram of the system.

This method has been shown analytically to determine thresholds or phase diagrams for a large class of mean field models, including automata, nonsymmetric spin glasses, neural networks, and simple mean field ferromagnets. ${ }^{(5-8)}$ A numerical study ${ }^{(6)}$ has also been carried out on the three-dimensional Ising model. For pure ferromagnetic couplings, the method gave the correct Curie temperature, whereas in the spin-glass case, the distance appeared to become nonzero at a significantly higher temperature than that usually interpreted as the spin-glass transition, but close to the temperature where nonexponential relaxation is believed to start.

Since there does not exist a general theory ${ }^{(9)}$ showing that this method is a reliable probe of phase diagrams, results obtained should be regarded more as dynamical properties than true equilibrium features of the system. Nevertheless, it is tempting to see what this dynamical approach gives in problems for which existing methods do not give a clear and accurate determination of the phase diagram.

The main goal of the present paper is to use this dynamical approach to investigate the existence of a floating phase in the two-dimensional ANNNI model. ${ }^{(3,10-18)}$ In Section 2, we define the model and explain the dynamical method. Section 3 contains our results for the distance between two configurations measured on four sweeps through the phase diagramthree as a function of temperature at fixed $\kappa=-J_{2} / J_{1}$ and one as a function of $\kappa$ at fixed temperature. Here $J_{1}$ and $J_{2}$ are the nearest and the second next-nearest neighbor interactions, respectively. Increasing temperature for small $\kappa$, we find evidence for a direct transition from ferromagnetic-like behavior to a paramagnetic phase. On the other hand, for large $\kappa$, we find a rather large region between the locked (antiphase) phase and the paramagnetic phase. We feel that this region can be interpreted as the floating phase of the two-dimensional ANNNI model. In Section 4, we show the variation with time and initial conditions of the distance and of the survival probability, i.e., the probability that the two initial configurations are still different after a certain time, at a few selected points of the phase diagram. We find that each phase can be characterized by a different behavior in these quantities. 


\section{MODEL AND METHOD}

We consider an Ising model $\left(\sigma_{i j}= \pm 1\right)$ on a square lattice governed by the Hamiltonian

$$
\mathscr{H}=-J_{1} \sum_{i, j}\left(\sigma_{i, j} \sigma_{i+1, j}+\sigma_{i, j} \sigma_{i, j+1}\right)-J_{2} \sum_{i, j} \sigma_{i, j} \sigma_{i+2, j}
$$

where the sum runs over all lattice sites. The nearest neighbor interaction $J_{1}$ is ferromagnetic $\left(J_{1}>0\right)$, whereas $J_{2}$ is antiferromagnetic $\left(J_{2}<0\right)$. We define $\kappa$ in the standard way:

$$
\kappa=-J_{2} / J_{1}
$$

and will measure temperature $T$ in units of $J_{1}$ so that, effectively, $J_{1}=1$.

Our calculations are performed for finite lattices of linear dimension $L$ with the boundary conditions

$$
\sigma_{i, j}=\sigma_{i+L, j}=\sigma_{i, j+L}=\sigma_{i+L / 2, j+L / 2}
$$

Hence, a sample of linear size $L$ consists of

$$
N=\frac{1}{2} L^{2}
$$

different spins. The reasons for choosing these boundary conditions are partly historical-we wrote our first program that way-and partly convenience-it allows an easy vectorization of the program. However, we do not think that this choice has any effect on our results, because $L$ is always chosen to be a multiple of eight, so that the boundary conditions are compatible with both the ferromagnetic configurations, $(+++\cdots+++)$ and $(--\ldots-\ldots)$, that are the ground states for $0<\kappa<0.5$ and with the periodic antiphase configurations, e.g., $(++-\cdots++--)$ that constitute the ground states for $\kappa>0.5$.

The dynamical evolution of configurations is based on a heat bath Glauber relaxation. To update the spin $\sigma_{i j}$ during the time interval $t$ to $t+d t$, we compute its local field $h_{i j}(t)$ at time $t$ :

$$
\begin{aligned}
h_{i j}(t)= & J_{1}\left[\sigma_{i, j+1}(t)+\sigma_{i, j-1}(t)+\sigma_{i+1, j}(t)+\sigma_{i-1, j}(t)\right] \\
& +J_{2}\left[\sigma_{i+2, j}(t)+\sigma_{i-2, j}(t)\right]
\end{aligned}
$$

and then choose

$$
\sigma_{i j}(t+d t)= \begin{cases}+1 & \text { with probability } \frac{1}{2}+\frac{1}{2} \tanh \frac{h_{i j}(t)}{T} \\ -1 & \text { with probability } \frac{1}{2}-\frac{1}{2} \tanh \frac{h_{i j}(t)}{T}\end{cases}
$$


Usually, at each time step in a Monte Carlo calculation, one chooses a spin $\sigma_{i j}$ at random and updates it according to (5) and (6). This ensures detailed balance and that the correct equilibrium at temperature $T$ is approached asymptotically. For this procedure, it is natural to scale the time step as $d t=1 / N$, where $N$ is the number of spins. One can also (and should on a vector computer) update simultaneously several spins. This form of updating also satisfies detailed balance and therefore leads to the right equilibrium as long as one updates simultaneously only spins that do not interact directly. In the calculations presented below, we update simultaneously rows of spins along the diagonal, i.e., during the first time step we update simultaneously $\sigma_{11}, \sigma_{22}, \ldots, \sigma_{1 / 2 L, 1 / 2 L}$, then during the next step, we update $\sigma_{12}, \sigma_{23}, \ldots, \sigma_{1 / 2 L, 1 / 2 L+1}$, followed during the next time step by $\sigma_{13}, \sigma_{24}, \ldots, \sigma_{1 / 2 L, 1 / 2 L+2}$, and so on. Of course, if $n$ spins are updated during a single time step, one should scale the time step as $d t=n / N$.

All the calculations described in the present paper concern the comparison of two different initial configurations submitted to the same thermal noise. Let us now explain how this is done. ${ }^{(5)}$ We start with two different initial configurations $\left\{\sigma_{i j}(0)\right\}$ and $\left\{\tilde{\sigma}_{i j}(0)\right\}$. Assume that the two systems are in configurations $\left\{\sigma_{i j}(t)\right\}$ and $\left\{\tilde{\sigma}_{i j}(t)\right\}$ at time $t$ and that we want to update the spins $\sigma_{i j}$ and $\tilde{\sigma}_{i j}$ during the time interval $t, t+d t$. We first compute the local fields $h_{i, j}(t)$ and $\widetilde{h}_{i j}(t)$ by (5) for each spin in the configurations. A random number $z_{i j}$, uniformly distributed between 0 and 1 , is then chosen and both spins $\sigma_{i j}$ and $\tilde{\sigma}_{i j}$ are updated according to (6) using the same random number $z_{i j}$. Explicitly,

$$
\begin{aligned}
& \sigma_{i j}(t+d t)=\operatorname{sign}\left[\frac{1}{2}+\frac{1}{2} \tanh \left(\frac{h_{i j}(t)}{T}\right)-z_{i j}\right] \\
& \tilde{\sigma}_{i j}(t+d t)=\operatorname{sign}\left[\frac{1}{2}+\frac{1}{2} \tanh \left(\frac{\tilde{h}_{i j}(t)}{T}\right)-z_{i j}\right]
\end{aligned}
$$

The easiest way to compare two configurations is to define the distance $\Delta(t)$ between them by

$$
\Delta(t)=\frac{1}{4 N} \sum_{i j}\left[\sigma_{i j}(t)-\tilde{\sigma}_{i j}(t)\right]^{2}
$$

where the sum is over all lattice sites. Defined this way, $\Delta(t)$ is the fraction of spins that are different in the two configurations [i.e., such that $\left.\sigma_{i j}(t)=-\tilde{\sigma}_{i j}(t)\right]$. If $\Delta(t)$ tends to zero as $t$ tends to infinity, then the two configurations become identical as $t$ increases and the system forgets its initial condition. Usually, we repeated our calculations for many samples and averaged $\Delta(t)$ over all samples. 
If for any reason, e.g., finite-size effects, fluctuations, convergence to a paramagnetic phase, the two configurations $\left\{\sigma_{i j}(t)\right\}$ and $\left\{\tilde{\sigma}_{i j}(t)\right\}$ are identical at time $t$, they will remain identical for all later times, since the same $z_{i j}$ is used in (7a) and (7b). As a result, it is more convenient to measure the distance $A(t)$ only for those samples that have survived to time $t$, i.e., for which $\Delta(t) \neq 0$. To do so, we introduce ${ }^{(5)}$ the survival probability $P(t)$, defined to be the fraction of samples for which the two configurations $\left\{\sigma_{i j}(t)\right\}$ and $\left\{\tilde{\sigma}_{i j}(t)\right\}$ are still different at time $t$. We then define the distance $\langle D(t)\rangle$ by averaging over only those samples that have survived, i.e.,

$$
\langle D(t)\rangle=\langle\mathcal{A}(t)\rangle / P(t)
$$

In the following two sections, we present curves of $\langle D(t)\rangle$ and $P(t)$ for various values of the coupling constants in (1). Of course using (9), it is possible to recover $\langle\Delta(t)\rangle$. The advantage of $\langle D(t)\rangle$ is that in most cases $P(t)$ and $\langle\Delta(t)\rangle$ depend on the initial conditions, e.g., on $\Delta(0)$, or on the fact that $\left\{\sigma_{i j}(0)\right\}$ or $\left\{\tilde{\sigma}_{i j}(0)\right\}$ is a ground state and on time, e.g., $P(t)$ tends to decrease with time due to finite-size effects or fluctuations, whereas $\langle D(t)\rangle$ does not suffer from these effects. On the other hand, since $P(t)$ decreases with time, the distance $D(t)$ is measured on smaller and smaller samples and therefore tends to fluctuate more and more as time increases.

\section{PHASE DIAGRAM}

The precise shape of the phase diagram of the ANNNI model varies from author to author. As an indication, we show the phase diagram calculated by Finel and de Fontaine ${ }^{(11)}$ in Fig. 1. It is a tremendous task to explore the whole phase diagram by any numerical method. We decided to limit ourselves to four sweeps through the phase diagram (see Fig. 1): three sweeps (denoted I, II, III) at fixed $\kappa(\kappa=0.0,0.2$, and 0.8$)$ and one sweep at a fixed temperature $(T=2.0)$.

Our calculations were performed for two lattice sizes $L=32$ and 64 . For $L=32$ we measured $\langle D(t)\rangle$ by averaging over 1000 samples at times $t=500$ and 1000 , while for $L=64$ we averaged $\langle D(t)\rangle$ over 200 samples at $t=1000$ and 2000 . In both cases, the initial configurations were chosen to be two ground states of the system: $(+++\cdots+++)$ and $(-\ldots \ldots--)$ for Figs. 2, 3, and 5 (for $\kappa<0.6$ ) and $(++--\cdots++--)$ and $(--++\cdots--++)$ for Figs. 4 and 5 (for $\kappa>0.6)$. The calculations could have been done starting with two random initial conditions (as in Section 4), but this would have required more iterations, since with random initial conditions equilibrium takes longer to 


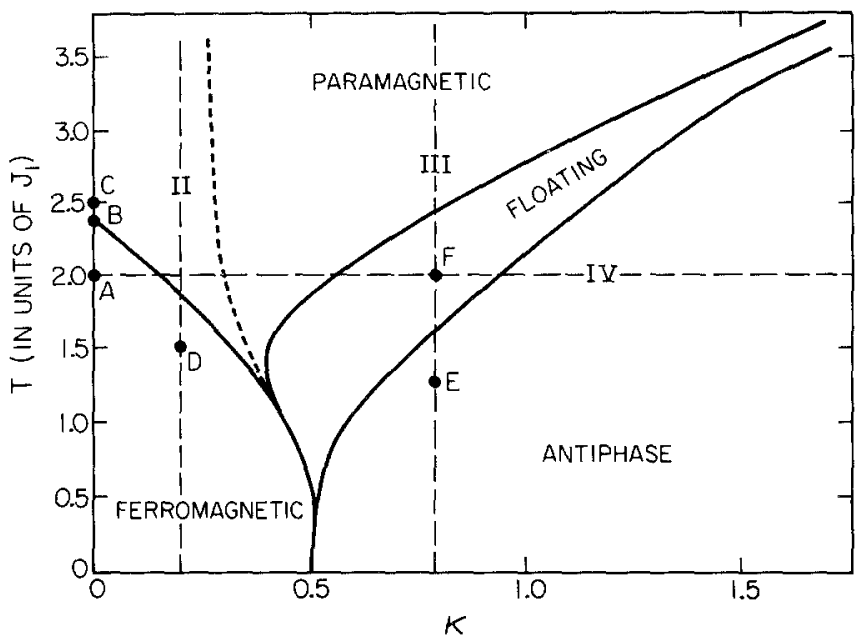

Fig. 1. Schematic representation of the phase diagram of the two-dimensional ANNNI model (after Finel and de Fontaine ${ }^{(11)}$ ). (- - Our sweeps II, III, and IV (sweep I is along the $\kappa=0$ axis) as well as the special points, marked $A-F$, discussed in Section 4. (- -) The estimated ${ }^{(11,13)}$ position of the disorder line.

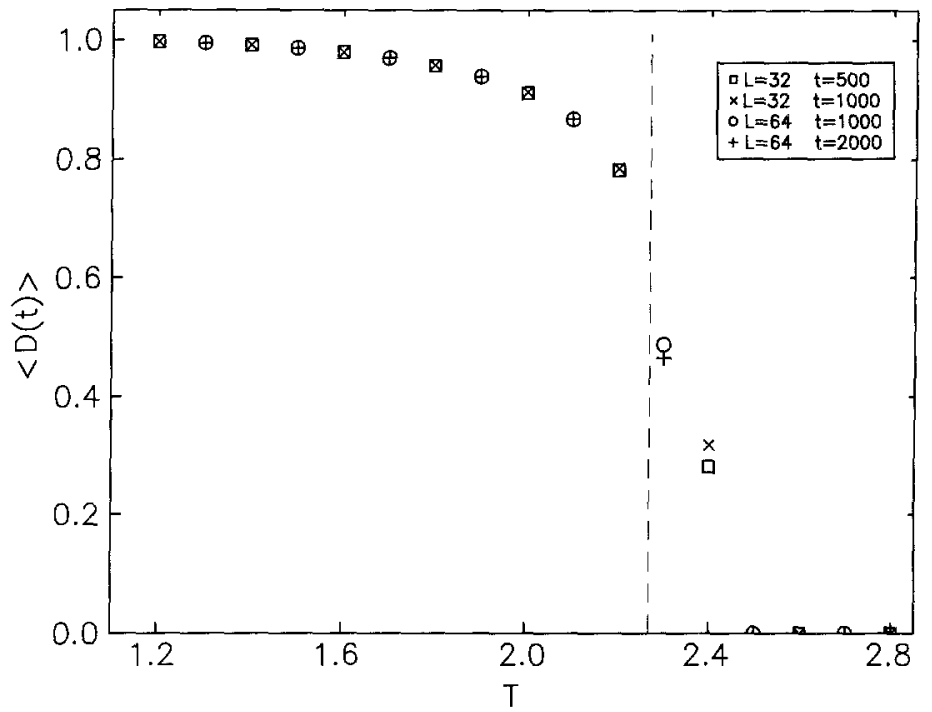

Fig. 2. Variation of $\langle D(t)\rangle$ as a function of temperature for $\kappa=0.0$ (sweep I). The broken vertical line marks the exactly known value of $T_{c}$. Note that for $T<2.2$, the symbols for different times and the same lattice superimpose, indicating the establishment of equilibrium. 


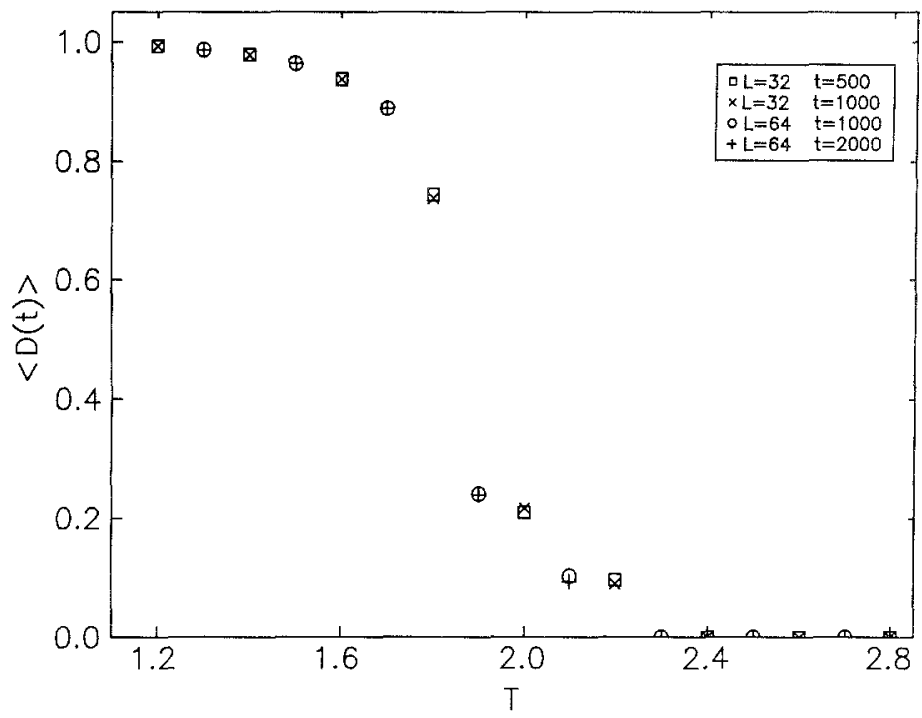

Fig. 3. Variation of $\langle D(t)\rangle$ as a function of temperature for $\kappa=0.2$ (sweep II).

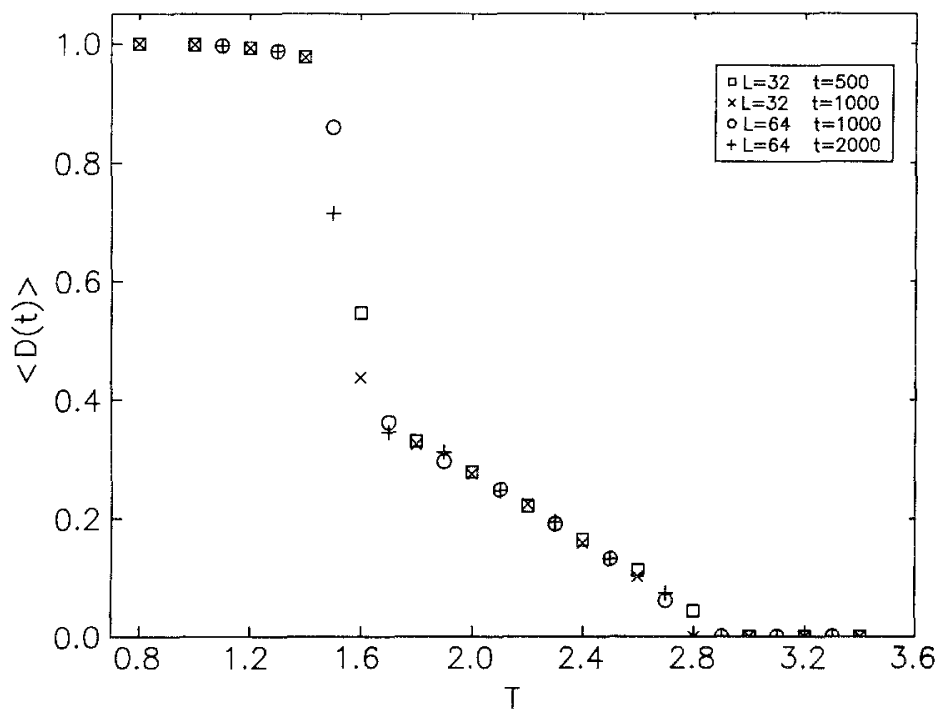

Fig. 4. Variation of $\langle D(t)\rangle$ as a function of temperature for $\kappa=0.8$ (sweep III). 
be established at low temperatures. However, the results would be very similar.

The behavior of $\langle D(t)\rangle$ for the standard two-dimensional ferromagnetic Ising model $(\kappa=0.0)$ is illustrated in Fig. 2. We see that equilibrium is reached at all temperatures except in the critical region, where finite-size and finite-time effects can be seen.

In Fig. 3, we see a rather similar situation for $\kappa=0.2$. There is clearly a transition at $T \approx 2.0$, with finite-size effects again apparent in the critical region. On the other hand, Fig. 4 for $\kappa=0.8$ presents a very different picture. We see evidence for two transitions: one at $T_{1} \approx 1.6$ and the other at $T_{2} \approx 2.6$. The low-temperature phase is the antiphase $(++--\cdots++--)$, while the phase $T_{1}<T<T_{2}$ seems to be the floating phase of the two-dimensional ANNNI model. In Section 4, we will see that the dynamical characteristics of $\langle D(t)\rangle$ and $P(t)$ are rather different in this intermediate phase from those seen in the antiphase or a simple paramagnetic phase.

Comparing our approach to other determinations of the phase diagram of the ANNNI model, we find that all the previous works ${ }^{(11-13)}$ would agree with our results for sweeps I and II (Figs. 2 and 3). However, for sweep III, which is of course the most interesting one, since it is an attempt to see the floating phase, our $T_{1}$ and $T_{2}$ agree rather well with the

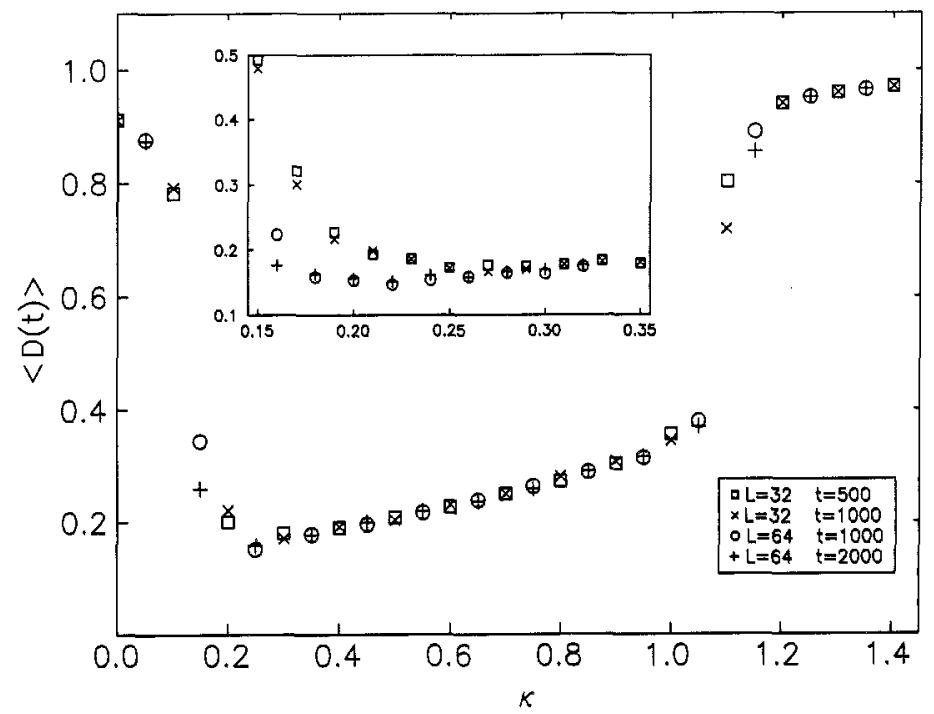

Fig. 5. Variation of $\langle D(t)\rangle$ as a function of $\kappa$ for $T=2.0$ (sweep IV). Inset: Enlargement of the range $0.15<\kappa<0.35$. 
work by Finel and de Fontaine, ${ }^{(11)}$ but are significantly higher than the transition temperatures estimated elsewhere. ${ }^{(12,13)}$

Figure 5 shows the results of our sweep in $\kappa$ at fixed temperature, $T=2.0$. We clearly see the transition from the floating phase to the antiphase at $\kappa \approx 1.2$. However, on the basis of these results, it is hard to say whether there is a direct transition from the ferromagnetic phase to the floating phase or if a narrow paramagnetic phase exists between the ferromagnetic and floating phases. Enlarging the scale (see inset) shows that the floating phase appears to exist down to at least $\kappa=0.3$, which is lower than all previous estimates. ${ }^{(1-13)}$ However, even on this scale, our results are not accurate enough to tell whether the finite-size and finite-time effects we see for $0.15<\kappa<0.3$ are due to two transitions separated by a narrow paramagnetic phase between or a single transition. It would be interesting to improve our results to obtain better evidence of a paramagnetic phase surrounding a disorder line ${ }^{(11,13)}$ extending down to the multicritical point at $T=0, \kappa=0.5$.

\section{TIME DEPENDENCES}

We now show that the time dependence of our two quantities of interest-the survival probability $P(t)$ and the averaged distance $\langle D(t)\rangle$-is rather different in the various phases. In Section 3 , we choose ground states for initial configurations to minimize the time it took to reach equilibrium. The time effects in which we are now interested are more easily seen if we choose our two initial configurations at random. We consider two cases:

IC1: $\sigma_{i j}(0)$ random with $\tilde{\sigma}_{i j}(0)=-\sigma_{i j}(0)$.

IC2: $\sigma_{i j}(0)$ and $\tilde{\sigma}_{i j}(0)$ both random and uncorrelated.

The system sizes ( $L=32$ and 64), the number of samples (1000 and 200), and the maximum times $(t=1000$ and 2000) are the same as in Section 3.

To establish some characteristic "benchmarks," it is useful to first consider the behavior of $P(t)$ and $\langle D(t)\rangle$ for the usual two-dimensional ferromagnetic Ising model $(\kappa=0)$. The time dependence of $P(t)$ and $\langle D(t)\rangle$ for initial conditions IC1 at temperatures $T=2.0<T_{c}, T=$ $T_{c}=2.269185 \ldots$, and $T=2.5>T_{c}$ are illustrated in Figs. 6a and 6b. The effect of changing the initial condition to IC2 is shown in Fig. 7 for $L=32$.

From these figures, we see that for $T<T_{c}$ the survival probability has a nonzero long-time limit, which does not appear to depend on the size. However, the time taken to reach this limit does depend on both the size and the initial conditions. (Indeed, for $L=64$, the long-time limit has not 

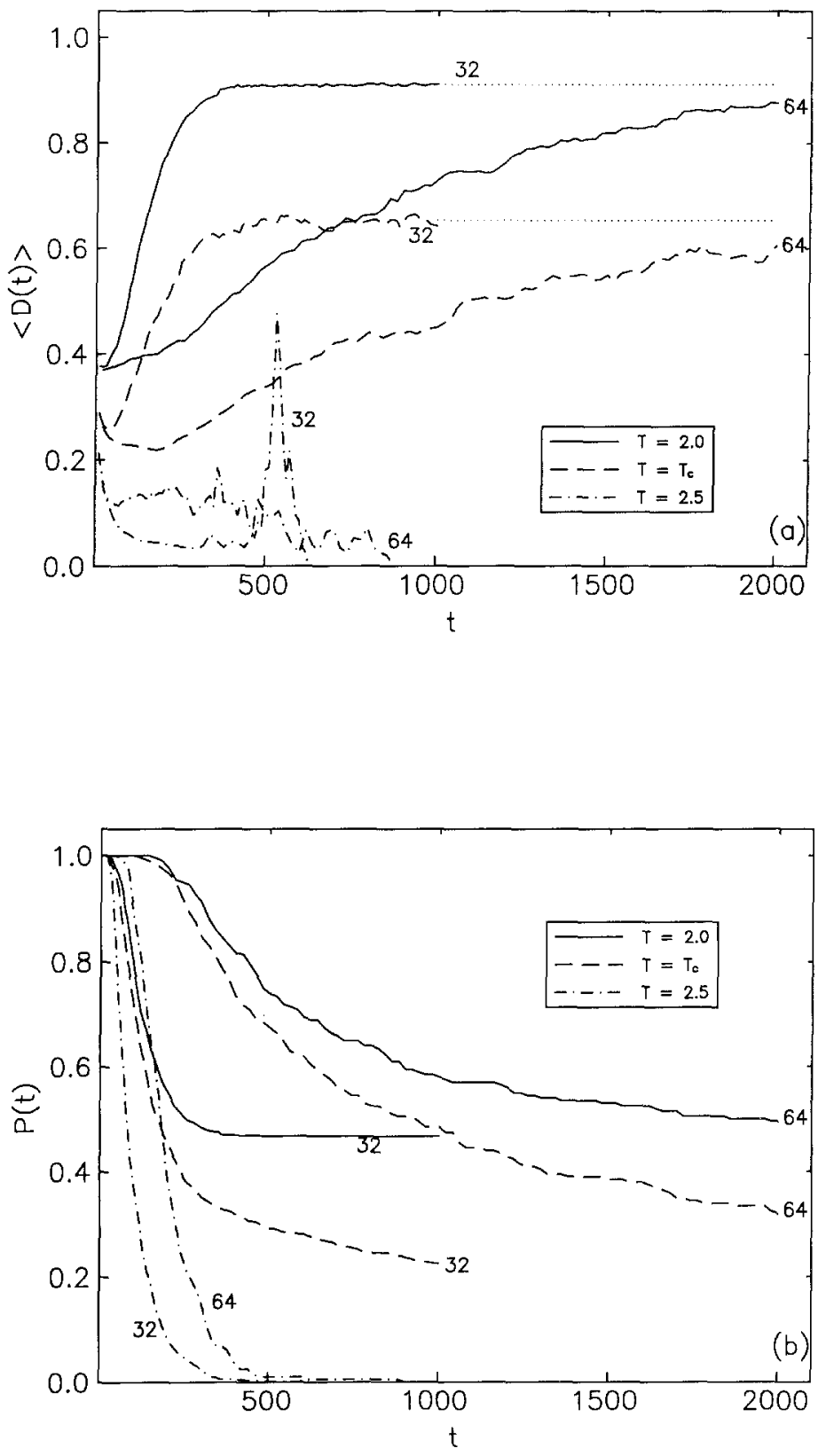

Fig. 6. Time variation of (a) $\langle D(t)\rangle$ and (b) $P(t)$ for initial conditions IC1 and $\kappa=0.0$. Note that for $L=32$, the iteration terminated at $t=1000$; the dotted lines for later time have been added only as a guide. 

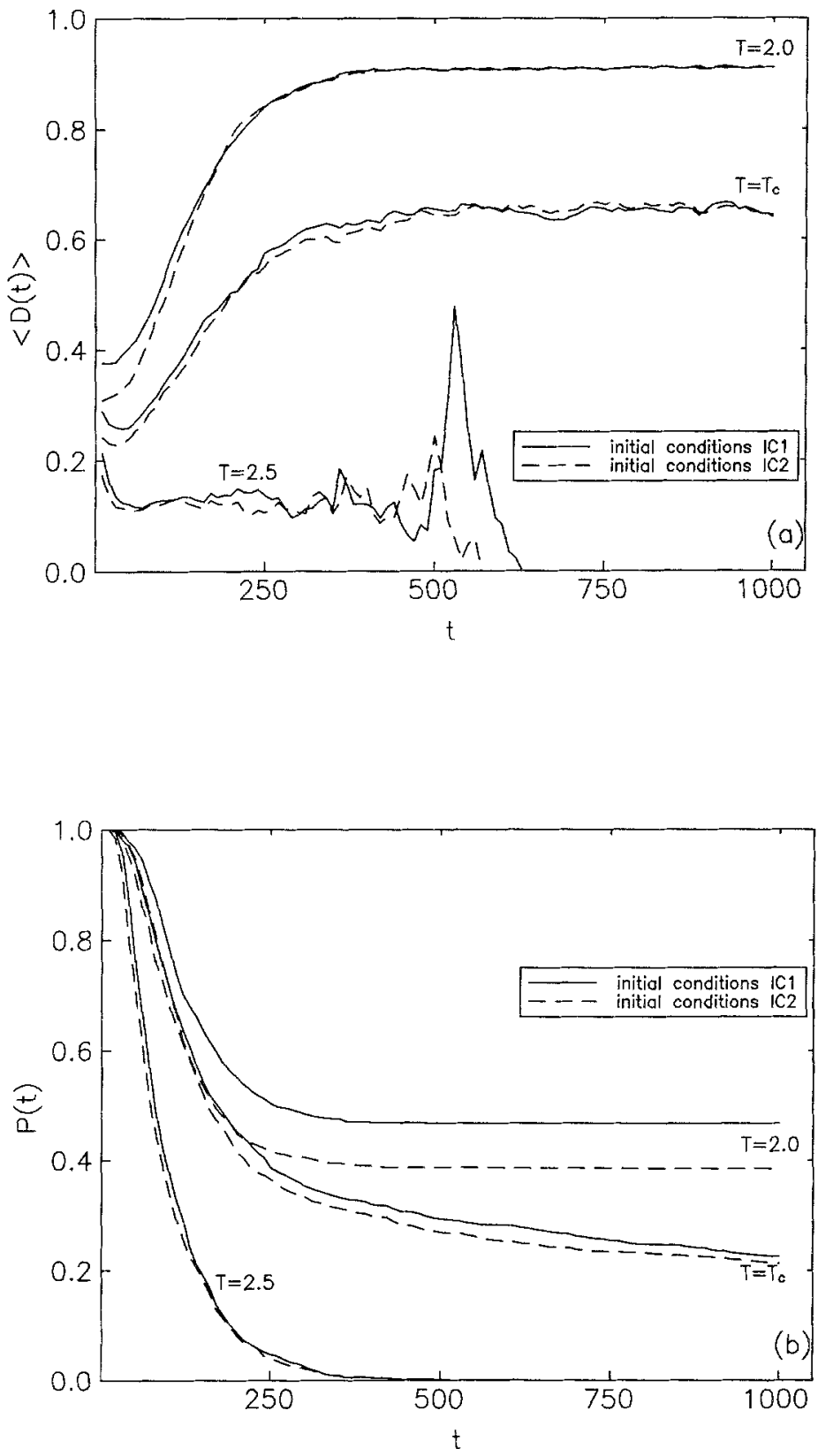

Fig. 7. Effect of initial conditions on the time variation of (a) $\langle D(t)\rangle$ and (b) $P(t)$ for $\kappa=0.0$ and $L=32$. 
yet been reached.) These two effects are easy to understand: the larger the system is, the longer it takes to eliminate the defects (domain walls) and changing the initial conditions affects the probability that two configurations fall into the same valley.

Turning now to the behavior of the distance $\langle D(t)\rangle$, we observe that, for $T<T_{c},\langle D(t)\rangle$ has a finite limit, which depends on neither $L$ nor on the initial condition. ${ }^{4}$ This behavior is also understandable: $\langle D(t)\rangle$ is the distance between the two valleys of the pure equilibrium phases. It is interesting to notice that $\langle D(t)\rangle$ changes with time on a time scale comparable to that of $P(t)$.

At $T=T_{c}$, the survival probability decreases to zero with time with a time scale that increases with $L$, as can be expected at a critical point. On the other hand, the distance $\langle D(t)\rangle$ appears to have a nonzero limit which decreases with $L$. For a model with only ferromagnetic interactions one can show ${ }^{(19)}$ that $\langle D(t)\rangle$ tends to the magnetization as $t$ tends to infinity and $L$ tends to infinity. Hence one expects $\lim _{t \rightarrow \infty}\langle D(t)\rangle$ to decrease like $L^{-\beta / v}$, where $\beta$ and $v$ have their usual definitions. For the two twodimensional Ising model, $\beta=1 / 8$ and $v=1$, so that the decay with $L$ is rather slow.

Finally, for $T>T_{c}$, we observe that the survival probability goes to zero very rapidly, while $\langle D(t)\rangle$ has a finite long-time limit, which decreases quickly with $L$. However, as soon as $P(t)$ is very small, $\langle D(t)\rangle$ starts to fluctuate considerably because of the small number of samples.

Similar results for $\kappa \neq 0$ are presented in Figs. 8-10. In Fig. 8, $\kappa=0.2$ and $T=1.5$, and we expect (see Fig. 1) that the system is in the ferromagnetic phase. We do see, indeed, that the time dependence is very similar to that found in the ferromagnetic phase of the simple Ising model.

In Fig. $9, \kappa=0.8$ and $T=1.3$, and we expect the system to be in the antiphase $(++--\cdots++--)$. Again the behavior is similar to that seen in Figs. 6 and 7 with $T<T_{c}$, except that the time scale is longer and the system still far from equilibrium. In addition, we would expect a further small difference with Fig. 8, since there are now four valleys. Consequently, $\langle D(t)\rangle$ should depend on the initial condition, since the probability of falling into two opposite valleys has no reason to be the same for different initial conditions.

Finally, in Fig. 10, we show results for $\kappa=0.8$ and $T=2.0$. This point is in the middle of the intermediate phase detected in Fig. 4 (recall also Fig. 5). We see that $P(t)$ decreases (at least for $L=32$ ) rather slowly with time, while $\langle D(t)\rangle$ reaches a long-time limit very quickly. It also appears

\footnotetext{
${ }^{4}$ Although for $L=64$ the limit is still not apparent after 2000 steps, we have checked that smaller sizes converge to the same limit as shown for $L=32$.
} 


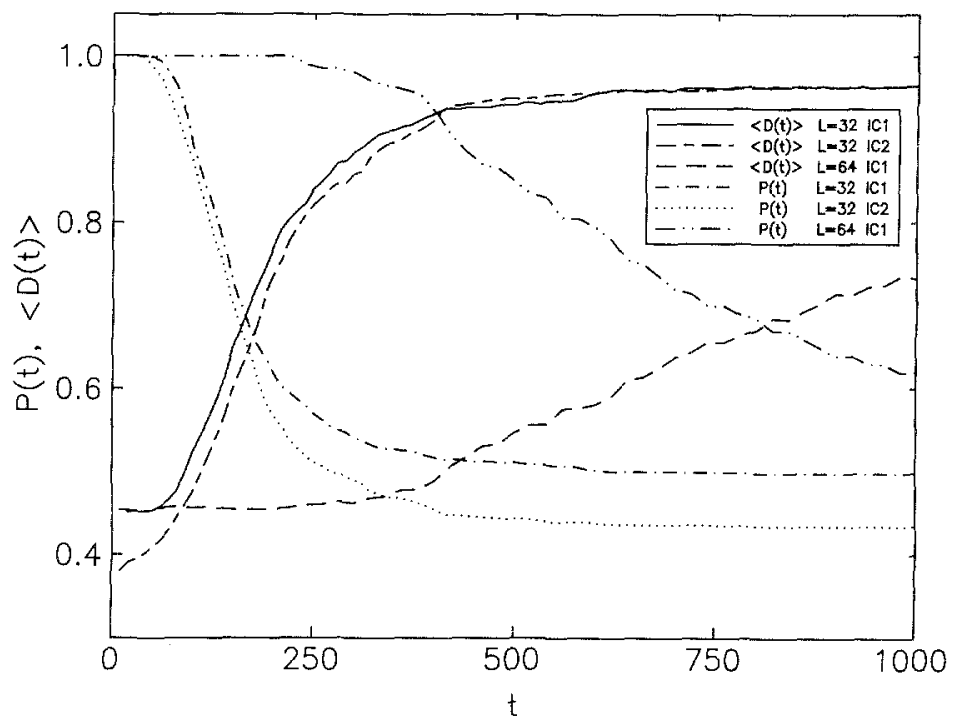

Fig. 8. Time variation of $\langle D(t)\rangle$ and $P(t)$ for $\kappa=0.2$ and $T=1.5$.

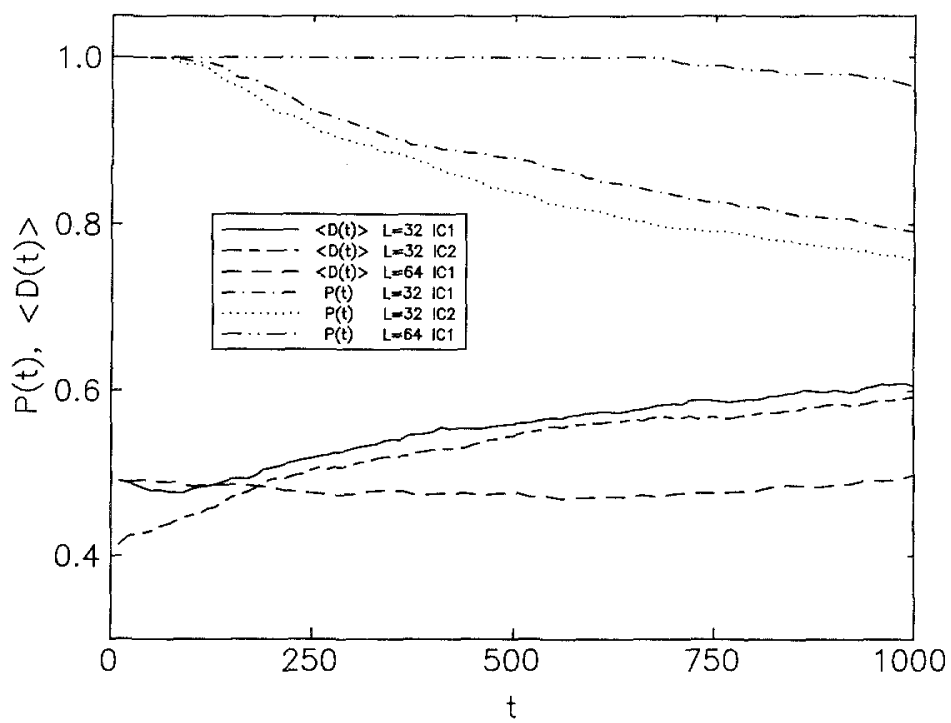

Fig. 9. Time variation of $\langle D(t)\rangle$ and $P(t)$ for $\kappa=0.8$ and $T=1.3$. 


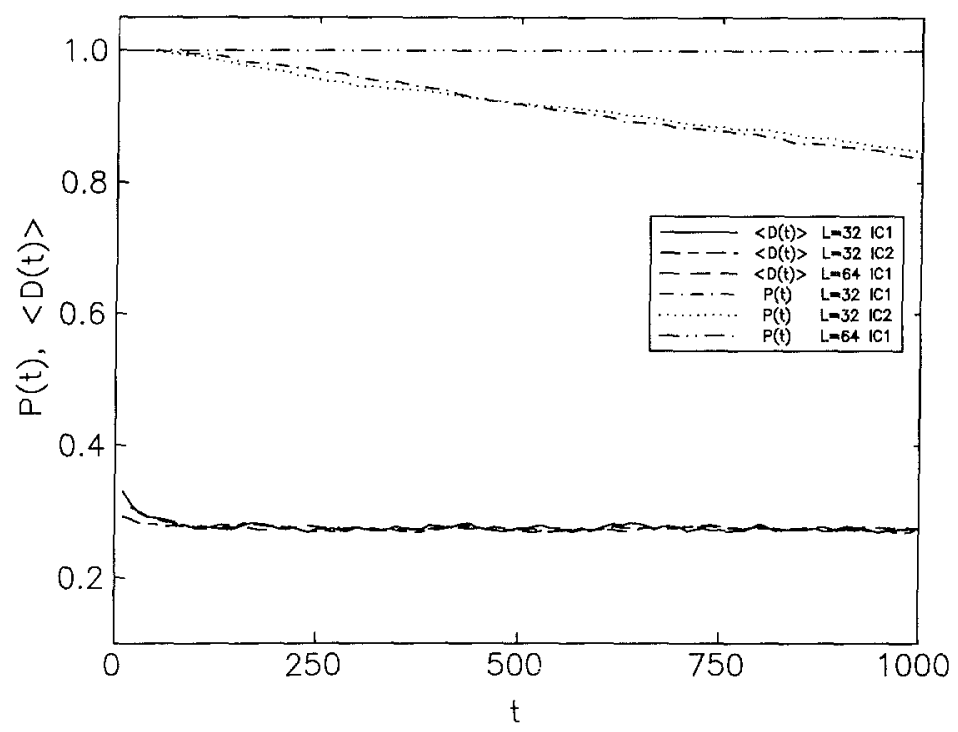

Fig. 10. Time variation of $\langle D(t)\rangle$ and $P(t)$ for $\kappa=0.8$ and $T=2.0$.

that this limit does not depend on $L$. We think that the fact that $\langle D(t)\rangle$ reaches its long-time limit so quickly is due to the fact that the surface tension is zero in the floating phase.

In summary: the various phases of the ANNNI model correlate with significantly different behavior of $P(t)$ and $\langle D(t)\rangle$ as functions of $t$ :

1. Ferromagnetic phase: $P(t)$ has a finite limit as $t$ goes to infinity (at least if one does not wait for times that increase exponentially with $L$ that allow jumps from the plus phase to the minus phase). $\langle D(t)\rangle$ varies slowly with time [on the same time scale as $P(t)$ ] and has a finite limit independent of $L$.

2. Critical point: $P(t)$ decreases to zero on a time scale that depends on $L$. The $\langle D(t)\rangle$ has a finite long-time limit, which decreases with $L$.

3. Paramagnetic phase: $P(t)$ decreases quickly to zero. $\langle D(t)\rangle$ has a finite limit, which decreases rapidly with $L$.

4. Floating phase: $P(t)$ decreases (probably to zero) on time scales that depend on $L$. The $\langle D(t)\rangle$ has a finite, long-time limit, which is reached quickly and does not depend on $L$. 


\section{CONCLUSION}

The main result of the present work could be summarized by Fig. 4 or Fig. 5. By monitoring the distance between two configurations submitted to the same thermal noise, we have found evidence for a floating phase between the antiphase $(++--\cdots++--)$ and the paramagnetic phase of the two-dimensional ANNNI model. However, the extent of this phase is rather larger than that found in previous calculations.

As yet, the dynamical approach used in this paper has not been tested on many systems. Consequently, it is difficult to judge whether our results are just dynamical effects or reliable predictions of the phase diagram at thermal equilibrium. Nevertheless, the results reported here suggest that further experimentation with and analysis of the method would be worthwhile and could establish the method as a reliable probe of equilibrium phases.

\section{ACKNOWLEDGMENTS}

This research was supported at the Institute for Theoretical Physics, Santa Barbara, by the National Science Foundation under grant PHY82-17853, supplemented by funds from the National Aeronautics and Space Administration.

\section{REFERENCES}

1. K. Binder and A. P. Young, Rev. Mod. Phys. 58:801 (1986).

2. D. Chowdhury, Spin Glasses and Other Frustrated Systems (World Scientific, Singapore, 1986).

3. W. Selke and M. E. Fisher, Z. Physik B 40:71 (1980).

4. W. Selke, A multitude of phases in the ANNNI model, preprint (1987).

5. B. Derrida and G. Weisbuch, Europhys. Lett. 4:657 (1987).

6. B. Derrida and G. Weisbuch, J. Phys. (Paris) 47:1297 (1986); B. Derrida, J. Phys. A 20:L721 (1987)

7. K. E. Kurten, Critical phenomena in model neural networks, Preprint (1987)

8. B. Derrida and O. Golinelli, preprint, 1988.

9. O. Martin, J. Stat. Phys. $41: 249$ (1985).

10. J. Villain and P. Bak, J. Phys. (Paris) 42:657 (1982).

11. A. Finel and D. de Fontaine, J. Stat. Phys. 43:645 (1986).

12. M. A. S. Saqi and D. S. McKenzie, J. Phys. A 20:471 (1987).

13. P. D. Beale, P. M. Duxbury, and J. Yeomans, Phys. Rev. B 31:7166 (1985).

14. M. N. Barber and W. Selke, J. Phys. A 15:2617 (1982).

15. P. M. Duxbury and M. N. Barber, J. Phys. A 15:3219 (1982).

16. J. Oitmaa, J. Phys. A 18:365 (1985).

17. T. Ala-Nissila, J. D. Gunton, and K. Kaski, Phys. Rev. B 33:7583 (1986).

18. T. Ala-Nissila, J. Amon, and J. D. Gunton, J. Phys. A 19:L41 (1986).

19. J. L. Lebowitz, private communication. 